\title{
Muscle Carnosine in Experimental Autoimmune Encephalomyelitis and Multiple Sclerosis
}

\author{
Charly Keytsman ${ }^{1}$, Laura Blancquaert ${ }^{2}$, Inez Wens ${ }^{1}$, Maarten Missine ${ }^{2}$, Pieter \\ Van Noten ${ }^{1}$, Frank Vandenabeele ${ }^{1}$, Wim Derave ${ }^{2}$, Bert 0 Eijnde ${ }^{1}$
}

(1) REVAL Rehabilitation Research Center, BIOMED Biomedical Research Institute, Faculty of Medicine and Life Sciences, Hasselt University, Agoralaan Building A, Diepenbeek, Belgium

(2) Department of Movement and Sports Sciences, Ghent University, Ghent, Belgium.

Corresponding author:

Charly Keytsman

Agoralaan Building A, B-3590 Diepenbeek, Belgium

Email: charly.keytsman@uhasselt.be;

Tel: +32477/61 1122 


\section{ABSTRACT}

Background. Muscle carnosine is related to contractile function ( $\mathrm{Ca}^{++}$handling) and buffering of exercise-induced acidosis. As these muscular functions are altered in Multiple Sclerosis (MS) it is relevant to understand muscle carnosine levels in MS.

Methods. Tibialis anterior muscle carnosine was measured in an animal MS model (EAE, experimental autoimmune encephalomyelitis, $n=40)$ and controls ( $\mathrm{CON}, \mathrm{n}=40)$ before and after exercise training

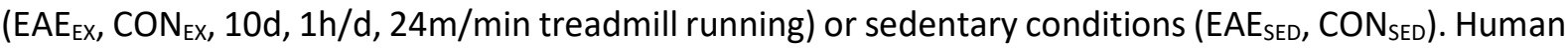
$m$. vastus lateralis carnosine of healthy controls $(H C, n=22)$ and MS patients $(n=24)$ were measured.

Results. EAE muscle carnosine levels were decreased $(p<0.0001)$ by $\sim 40 \%$ to $\sim 64 \%$ at $10 d$ and $17 d$ following EAE induction (respectively) regardless of exercise $(p=0.823)$. Similarly, human MS muscle carnosine levels were decreased $(-25 \%, p=0.03)$.

Conclusion. Muscle carnosine concentrations in an animal MS model and MS patients are substantially reduced. In EAE exercise therapy does not restore this.

Key words: experimental autoimmune encephalomyelitis; multiple sclerosis; carnosine; rehabilitation, muscle; neuromuscular 


\section{INTRODUCTION}

Multiple sclerosis (MS) is a neurodegenerative autoimmune disease of the central nervous system (Pugliatti et al., 2006), in which inflammatory and demyelination processes also result in physical inactivity, decreased exercise capacity, excessive (post-exercise) fatigue and reduced muscle contractile function (Dalgas et al., 2008; Savci et al., 2005). These frequently occurring secondary symptoms substantially affect various daily life activities, ultimately leading to an impaired quality of life (Ellis and Motl, 2013). Central mechanisms (de Haan et al., 2000; Kent-Braun et al., 1997; Rice et al., 1992; Sharma et al., 1995; van der Kamp et al., 1991) such as reduced motor firing rates, impaired motor unit recruitment and increased central motor conduction time clearly contribute to the above described disuse-related physiological profile ( $\mathrm{Ng}$ and Kent-Braun, 1997). A portion of the neuromuscular dysfunction in MS however, probably also resides within the skeletal muscle (de Haan et al., 2000; Garner and Widrick, 2003; Kent-Braun et al., 1997; Kent-Braun et al., 1994; Rice et al., 1992; Sharma et al., 1995; Wens et al., 2014).

We, and others, already reported altered muscle fiber composition (shift to glycolytic fibers), disturbed muscle contractile characteristics and cross-bridge ( $\mathrm{Ca}^{2+}$ handling) abnormalities in muscles of MS patients (de Haan et al., 2000; Garner and Widrick, 2003; Wens et al., 2014). Furthermore, reduced Krebs cycle and complex I and II activities (Kent-Braun et al., 1997; Kumleh et al., 2006), overproduction of reactive oxygen species (ROS) (Haider et al., 2011), increased basal AMP-activated protein kinase phosphorylation (Hansen et al., 2015) and delayed phosphocreatine resynthesis after exercise (Campbell et al., 2013; Hansen et al., 2015; Kent-Braun et al., 1997; Kent-Braun et al., 1994; Kumleh et al., 2006) suggest muscular mitochondrial dysfunction in MS and higher basal and exercise-related energy expenditure. In keeping with this, increased intramyocellular lactate accumulation, leading to increased basal serum lactate concentrations (Amorini et al., 2014), have been shown to cause greater (perceived) muscle fatigue in MS patients. It thus seems that MS patients do not only exhibit central neurological abnormalities but also impairments in both muscular contraction mechanisms and energy 
supply. In keeping with this, exercise rehabilitation therapy, that amongst others induces muscular acidosis, has become an important part of overall MS treatment (Motl and Gosney, 2008).

So far, small but significant improvements in exercise capacity and muscle contractile characteristics following various types (modality, frequency) of short to longer term regular exercise therapy have been reported in both a frequently used animal MS model, notably Experimental Autoimmune Encephalomyelitis (EAE)(Wens et al., 2015b) and in MS (Kjolhede et al., 2012; Motl et al., 2008; Petajan et al., 1996; Sallis et al., 1986; Schulz et al., 2004; Stuifbergen et al., 2006). In an attempt to further improve muscle contractile and energy metabolic responses to exercise, our laboratory recently performed high(er) intensity training studies in MS showing substantially improved (+25-50\%) muscle strength and exercise capacity following 8-12w of exercise (Wens et al., 2015a; Wens et al., 2015b). Although promising, most MS subjects however reported higher post-exercise muscle fatigue and overall perceived exertion rates (BORG: $14.7 \pm 1.5$ vs. $12.7 \pm 1.3$ ) compared to regular intensity training (Wens et al., 2014). Consequently, any strategy that attenuates this may further improve therapy outcome.

Carnosine, a dipeptide composed of $\beta$-alanine and L-histidine, is found in high concentrations in mammalian skeletal muscle (Boldyrev et al., 2013). Together with anserine or ophidine/balenine, the methylated analogs that possess the same bioactivity and are exclusively found in animals, carnosine forms the histidine-containing dipeptides (HCD). The physiological role of carnosine is related to contractile function in general and more specific to $\mathrm{Ca}^{++}$handling (Derave et al., 2010). Interestingly, carnosine has also been shown to buffer exercise-induced acidosis (Derave et al., 2010), to affect mitochondrial respiration (Boldyrev, 2007) and to protect against exercise-induced oxidative stress (Dawson et al., 2002). The functional role of carnosine in skeletal muscle is thus closely related to several of the above described muscle alterations seen in MS. So far, altered tissue carnosine concentrations were shown in other neurological diseases such as Amyotrophic Lateral Sclerosis (ALS) 
(Stuerenburg and Kunze, 1999) and Parkinson's Disease (Bellia et al., 2014; Boldyrev et al., 2013). Moreover, reduced serum carnosinase activity was already found in patients with MS (Wassif et al., 1994), indicating that several of the above described muscle alterations like impaired exercise capacity, excessive (post-exercise) fatigue and reduced muscle contractile function in MS (Dalgas et al., 2008; Savci et al., 2005; Wens et al., 2014) may, in part, be related to reduced muscle carnosine levels. This, however, has not been investigated in animal MS models and/or MS yet. Finally, it is important to note that although the overall impact of exercise on muscle carnosine content in healthy controls and disease is contradictory (Baguet et al., 2011; Derave et al., 2007; Kendrick et al., 2008; Kendrick et al., 2009; Mannion et al., 1994; Suzuki, 2004), increased muscle carnosine has been shown to reduce exercise-induced fatigue and hereby improve exercise performance during high-intensity intermittent exercise (1-4min) (Blancquaert et al., 2015; Derave et al., 2010; Derave et al., 2007). Therefore, the first aim of this study is to investigate muscle carnosine levels in both EAE animals and MS patients. We hypothesize that muscle carnosine concentrations in EAE and MS are reduced compared to healthy controls. Secondly, the effect of exercise on muscle carnosine levels in EAE rats is explored.

\section{METHODS}

Muscle carnosine content was measured in both an animal MS model, notably Experimental Autoimmune Encephalomyelitis (EAE, Part I), and MS patients (Part II). The analyzed muscle samples originate from studies that were previously performed and published by our laboratories (Keytsman et al., 2017; Wens et al., 2015b).

\subsection{Part I}

In a first phase, rodent muscle carnosine and anserine levels were investigated using the EAE animal MS model (Constantinescu et al., 2011). Briefly, this model is characterized by the induction of EAE on 
day 0 , an inflammatory period with no clinical symptoms (day 0 to day 10 ) and gradual hindquarter paralysis (day 11 to day 14 ) followed by almost full recovery (day 15 to day 17$)$.

\subsubsection{Animals}

Eighty female Lewis rats (age 6-7 weeks, body weight 100-120 g, Harlan CPB, Zeist, The Netherlands) were individually housed $\left(12 \mathrm{~h} / 12 \mathrm{~h}\right.$ light/dark cycle; temperature of $22^{\circ} \mathrm{C}$; relative humidity of $\left.22-24 \%\right)$ in the animal facilities at Hasselt University. Rats were fed ad libitum with water and normal rat pellets (Carfil RN-01-K12, Harlan). The animal Ethics Committee of Hasselt University approved the study protocol that complied with the national/European legislation and the National Research Council's guide for the care and use of laboratory animals.

\subsubsection{Study design}

Following acclimatization and adaptation (day -21 to -15 ), animals were randomized into two subgroups: a sedentary group ( $S E D, n=40$ ) and an exercise group ( $E X, n=40)$. In order to induce comparable levels of stress, SED animals were seated on the stationary treadmill (1 hour) on a daily basis (day -14 to 0 ). Daily food intake and body weight were registered. At day 0, SED and EX groups were subdivided into a healthy control group $\left(\mathrm{CON}_{\mathrm{SED}} \mathrm{n}=20 ; \mathrm{CON}_{\mathrm{EX}} \mathrm{n}=20\right)$ and an EAE group (EAE $\mathrm{E}_{\mathrm{SED}}$ $n=20 ; E_{E x} n=20$ ). Briefly, $E A E$ was induced by a single percutaneous injection in both footpads $(100 \mu \mathrm{l} /$ foot) under isoflurane anesthesia and consisted, per animal, of $24 \mu \mathrm{l}$ purified myelin basic protein (MBP, $25 \mathrm{mg} / \mathrm{ml}$ ) in combination with $25 \mu \mathrm{l}$ 7RA heat-killed Mycobacterium Tuberculosis $(20 \mathrm{mg} / \mathrm{ml}$, Difco), $120 u ̀ l$ complete Freunds adjuvant (CFA, Difco) and $31 \mu \mathrm{l}$ phosphate-buffered saline (PBS) (Polfliet et al., 2002). Hereafter, EX rats exercised daily for 1 hour/day, during 10 consecutive days, until progressive hindquarter paralysis prevented this. Treadmill performance/intensity was 
visually monitored daily by the researchers and did not appear to be different between the control and EAE groups.

At day 10 (D10), just before onset of hindquarter paralysis, treadmill training was terminated and 10 animals of each group were evaluated. The remaining rats ( $n=10$ per group) endured hindquarter paralyses (EAE group, day $11-14$ ) and were evaluated after (almost full) recovery on day 17 (D17). All animals were anaesthetized using an intraperitoneal injection of pentobarbital sodium (5 mg $100 \mathrm{~g}-1$ BW) and $\mathrm{m}$. tibialis anterior (TA) of the right hind limb was dissected and freed of connective tissue and visible blood. The mid-part of each muscle was snap-frozen using liquid nitrogen, and stored at $80^{\circ} \mathrm{C}$ until further analysis were performed. All animals were sacrificed after muscle sampling.

Throughout the study course, animals were carefully monitored on daily basis. Animals did not display severe discomfort immediately following immunization. Paralysis and typical signs of EAE occurred 11 days post-immunization, which is the typical time frame for this animal model. If animals exhibited signs of severe pain (high clinical score for 2 consecutive days), distress, were unable to reach food or when humane endpoints were reached, euthanasia to relief pain was performed. However, none of the animals included in the study underwent euthanasia for one of those reasons.

\subsection{Part II}

\subsubsection{MS subjects}

Twenty-two healthy controls $(\mathrm{HC})$ and twenty-four patients with MS (aged >18 years; mean EDSS $3.1 \pm 1.5$, range $0 \rightarrow 6$, median 2.5 ) were included following written informed consent. Subjects were excluded if they participated in other studies, had (in case of MS) an acute exacerbation 6 months prior to the start of the study or had an EDSS score $>6$. Phenotypes of MS (RR, relapsing remitting; PP, primary progressive; SP, secondary progressive) and the duration of the disease were inventoried. The study was approved by the local Ethical Committee of the Jessa hospital and Hasselt University and 
complied with the Declaration of Helsinki. This study was registered at ClinicalTrials.gov (NCT02466165).

\subsubsection{Muscle biopsies}

Muscle biopsies were obtained from MS patients $(n=24)$ and HC $(n=22)$ from the middle part of the m. vastus lateralis (Bergström needle technique), by an experienced medical doctor. Biopsies were sampled from the weakest leg, as assessed by a preceding isometric muscle strength test performed on an isokinetic dynamometer (System 3, Biodex, ENRAF-NONIUS, New York, USA). The biopsied leg of HC was randomized, since muscle strength associated with each leg (left vs. right or dominant vs. nondominant) is equal in healthy persons (McCurdy and Langford, 2005; Siqueira et al., 2002). The sample was freed from connective tissue and snap-frozen with liquid nitrogen and stored at $-80^{\circ} \mathrm{C}$, until further analysis.

\subsection{Muscle dipeptide and free amino acid content}

Dipeptide and free amino acid concentrations were determined by high-performance liquid chromatography (HPLC), as previously described (Everaert et al., 2013). Muscle samples (15mg wet weight, WW) were deproteinized using $35 \%$ sulfosalicylic acid and centrifuged (5min, 16,000g). Deproteinized supernatant $(5 \mu \mathrm{l}$ sample) was mixed with AccQ Fluor Borate buffer $(75 \mu \mathrm{l})$ and reconstituted Fluor Reagent (20 $\mu$ l) from the AccQTag chemistry kit (Waters). The derivatized samples were applied to a Waters HPLC system comprised of an XBridge BEH column (4.6 $\times 150 \mathrm{~mm}, 2.5 \mu \mathrm{m})$ and fluorescence detector (excitation/emission wavelength: 250/395nm). Carnosine and anserine were assessed in Part I (animals) and carnosine, taurine, serine, glutamine and histidine were measured in Part II (human subjects). To compare muscle biopsy quality between healthy controls and MS, some reference muscle amino acid (serine, histidine and glutamine, in Part II) concentrations, 
expressed as total areas under the curve (tAUC), were compared between groups. In case of normal serine, histidine and glutamine concentrations, low carnosine concentrations could not be due to poor biopsy quality .

\subsection{Statistical Analysis}

All data were analyzed using SPPS v. 22.0 (IBM). Normality of data distribution was evaluated using the Shapiro-Wilk test. In animals, ANOVA was used to evaluate the (main and interaction) effect of 'group' (CON vs. EAE), 'activity' (SED vs. EX) and 'time' (D10 vs. D17) on carnosine and anserine concentrations and. For human analysis, group comparison was performed using unpaired student's t-tests in case of normality and non-parametric independent t-tests (Wilcoxon) otherwise. All data are presented as means $\pm S D$ and the threshold for statistical significance was set at $p<0.05$.

\section{RESULTS}

\subsection{Part I.}

\subsubsection{Rat muscle carnosine and anserine concentrations}

Muscle carnosine concentrations were significantly lower (main group effect, CON vs. EAE, $p<0.0001$ range: $-40 \% \rightarrow-64 \%$ ) in EAE compared to CON and D10 to D17(main time effect, D10 vs. D17, $p=0.005$, range: $-4 \% \rightarrow-46 \%$ )(Table 1). However, exercise had no influence on carnosine concentrations (main activity effect, SED vs. EX, $p=0.823)$. No interaction effects were found.

Muscle anserine was significantly higher in EAE compared to CON (main group effect, CON vs. EAE, $p=0000$, range: $+36 \% \rightarrow+40 \%)$. No main activity and time effects as well as interaction effects were detected. 
Table 1. Rat muscle carnosine and anserine concentrations.

\begin{tabular}{lccccccccc}
\hline & \multicolumn{2}{c}{ CONSED } & \multicolumn{2}{c}{ CON } & \multicolumn{3}{c}{ EAEX } & & \multicolumn{2}{c}{ EAE $_{\text {EX }}$} \\
& D10 & D17 & D10 & D17 & D10 & D17 & D10 & D17 \\
Carnosine & $2.5 \pm 0.6$ & $2.2 \pm 0.4$ & $2.5 \pm 0.6$ & $2.4 \pm 1.1$ & $1.5 \pm 0.6$ & $0.8 \pm 0.4$ & $1.4 \pm 0.3$ & $0.9 \pm 0.5$ \\
Anserine & $2.2 \pm 0.7$ & $2.5 \pm 0.4$ & $2 \pm 0.5$ & $2.3 \pm 1.0$ & $3 \pm 0.7$ & $3.4 \pm 1.0$ & $3.5 \pm 0.7$ & $2.9 \pm 0.6$ \\
\hline \hline
\end{tabular}

Data are expressed as means $\pm S D$ and represent rat $m$. tibialis anterior carnosine and anserine concentrations (mmol/kg WW) 10 (D10, n=10/group) and 17 (D17, n=10/group) days after acute experimental autoimmune

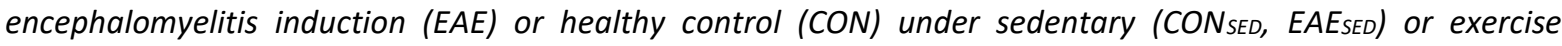
(treadmill running, $1 \mathrm{~h} / \mathrm{d}, 18 \mathrm{~m} / \mathrm{min}, C_{E X}, E A E_{E X}$ ) conditions. Main effects for group (CON vs. EAE, $p=0000$ ) and time (D10 vs. $D 17, p=0.005)$ were present for carnosine concentrations. For anserine concentrations, a main group effect (CON vs. EAE, $p=0000$ ) was found.

\subsection{Part II.}

\subsubsection{Subject characteristics}

Subject characteristics (Table 2) did not differ between HC and MS. Relapsing-remitting MS was diagnosed in 16 patients, whilst 6 patients suffered from primary-progressive MS and 2 patients from the secondary- progressive form. Mean disease duration was $13 \pm 8$ years (range $1 \rightarrow 26 y$ ).

Table 2. Baseline subject characteristics.

\begin{tabular}{lcc}
\hline & HC & MS \\
Age (years) & $48.7 \pm 11.8$ & $52.7 \pm 8.9$ \\
Gender (f/m) & $14 / 8$ & $13 / 11$ \\
Weight (kg) & $69.5 \pm 13.8$ & $71.6 \pm 12.4$ \\
Height (m) & $1.69 \pm 0.1$ & $1.71 \pm 0.1$ \\
BMI & $24.1 \pm 2.4$ & $24.5 \pm 3.5$ \\
Disease duration (years) & $/$ & $13 \pm 8$ \\
EDSS & $/$ & $3.1 \pm 1.5$ \\
\hline \hline
\end{tabular}

Data are expressed as means $\pm S D$ and represent subject characteristics (BMI: body mass index) of healthy controls (HC, $n=22)$ and MS patients $(n=24)$. EDSS, Expanded Disability Status Scale.

\subsubsection{Muscle carnosine and taurine content}

In MS, muscle carnosine $(3.8 \pm 1.2 \mathrm{mmol} / \mathrm{kg} W W$ vs. $2.9 \pm 1.3 \mathrm{mmol} / \mathrm{kg} \mathrm{WW}, \mathrm{p}=0.03)$ and muscle taurine

$(8.4 \pm 3.3 \mathrm{mmol} / \mathrm{kg} \mathrm{WW}$ vs. $6.5 \pm 2.9 \mathrm{mmol} / \mathrm{kg} \mathrm{WW}, \mathrm{p}=0.04)$ levels were lower compared to HC (Figure1). 
Total areas under the curve of muscle serine $(5.1 \pm 2.7$ vs. $4.3 \pm 1.1, p=0.12)$, histidine $(6.2 \pm 1.6$ vs. $5.5 \pm 1.5, p=0.07)$ and glutamine $(59.7 \pm 30.3$ vs. $61.1 \pm 28.6, p=0.43)$ did not differ between groups (data not shown).

Fig 1 Muscle carnosine and taurine content of healthy controls and multiple sclerosis
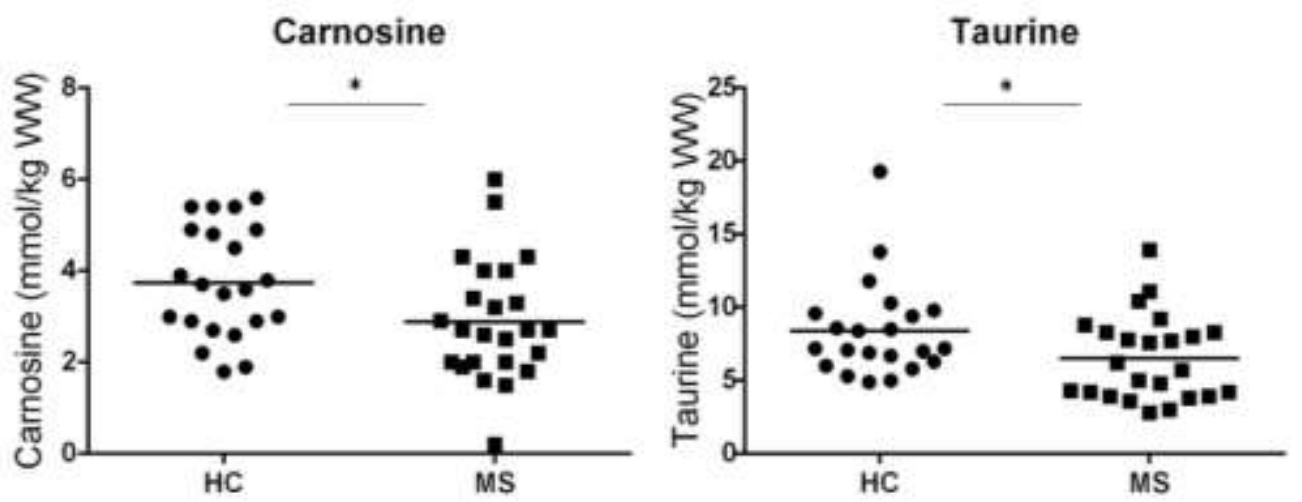

Data represent $\mathrm{m}$. vastus lateralis carnosine and taurine concentrations ( $\mathrm{mmol} / \mathrm{kg} \mathrm{WW}$ ) of healthy controls $(H C, n=22)$ and MS patients $(n=24)$. ${ }^{*} p<0.05$ between HC and MS.

\section{DISCUSSION}

Compared to healthy controls the present study clearly shows reduced muscle carnosine levels in both human MS and a frequently used animal MS model. Furthermore, in animals, exercise did not restore carnosine concentrations. In MS, lower muscle carnosine was paralleled by reduced taurine.

Literature indicates that carnosine metabolism could be altered in neuromuscular diseases. Wassif et al. (Wassif et al., 1994) already demonstrated reduced serum carnosinase activity (-49\%) in fresh blood samples from MS patients compared to healthy controls. Carnosinase causes hydrolysis of carnosine into $\beta$-alanine and L-histidine. This suggests alterations in carnosine metabolism in patients with MS. Stuerenburg (Stuerenburg and Kunze, 1999) investigated the carnosine content of skeletal muscles from patients with suggestive neuromuscular diseases and rats of various ages. Following stepwise regression modelling they reported that another neurological disease such as ALS was negatively correlated with muscle carnosine content and they suggested that this was caused by progressive 
denervation processes, as also seen in MS. Although altered tissue carnosine concentrations in other neuromuscular diseases such as ALS (Stuerenburg and Kunze, 1999) and Parkinson Disease (Bellia et al., 2014; Boldyrev et al., 2013) and reduced serum carnosinase activity in MS (Wassif et al., 1994) were already described, we are now the first to report substantially reduced muscle carnosine levels in an animal MS model and confirm this in MS patients.

Apart from reduced muscle carnosine in EAE we also detected increased muscle anserine concentrations. Interestingly anserine, the methylated form of carnosine, is exclusively found in animals where it possesses the same bioactivity as carnosine (Boldyrev et al., 2013). Hence, when carnosine decreases and anserine tends to increase (Table 1), the total amount of histidine-containing dipeptides (HCD) remains unchanged. In order to keep the total HCD concentration constant, it is possible that carnosine methyltransferase $(\mathrm{CMT})$ is upregulated following EAE induction, leading to enhanced muscle anserine levels. Interestingly, Drozak et al. (2015) recently molecularly identified UPF0586 protein C9orf41 as the mammalian carnosine-N-methyltransferase, responsible for anserine formation in rat muscle (Drozak et al., 2015). However, to date, no activity assay for CMT is available. Quantitative PCR could be an alternative to measure the effect of EAE on CMT gene expression, but this is not always in agreement with the amount of mRNA that is effectively translated into protein. In human subjects however, the methylated analog anserine is absent. Therefore, in humans a decrease in muscle carnosine content implicates an effective reduction of the total histidine-containing dipeptide store.

Although the exact underlying mechanisms remain unclear, several contributing factors may explain reduced carnosine stores in MS muscles. In MS, central mechanisms (de Haan et al., 2000; Kent-Braun et al., 1997; Rice et al., 1992; Sharma et al., 1995; van der Kamp et al., 1991), such as impaired motor unit recruitment and delayed conduction/reaction times induce a disuse-related physiological profile (Ng and Kent-Braun, 1997). In sedentary populations and in chronic disease (e.g. ALS, osteoarthritis), lower muscle carnosine levels (Stuerenburg and Kunze, 1999; Tallon et al., 2007) have been reported 
that might result from an inactivity related reduction in muscle protein content. However, in the present study muscle histidine, glutamine and serine, that are also prone to inactivity, were not affected in MS muscle samples. As such, we assume that the presented decrease in muscle carnosine content was not due to poor biopsy or muscle quality. Possibly, several intramyocellular dysfunctions that are associated with MS and relate to the physiological role(s) of carnosine in muscle (Boldyrev et al., 2013), could partly be related to the present findings. However, the exact effects of muscle carnosine on, amongst others, disturbed muscle contractile characteristics and cross-bridge $\left(\mathrm{Ca}^{2+}\right.$ handling) abnormalities (de Haan et al., 2000; Garner and Widrick, 2003; Wens et al., 2014), excessive exercise-induced acidosis (de Haan et al., 2000; Kent-Braun et al., 1997; Sharma et al., 1995) and mitochondrial dysfunction (Campbell et al., 2013; Hansen et al., 2015; Kent-Braun et al., 1997; KentBraun et al., 1994; Kumleh et al., 2006; Sharma et al., 1995) in MS have not been investigated yet.

The effect of exercise therapy on muscle carnosine content is not fully clear. With the exception of Suzuki et al. (Suzuki, 2004), who detected increased carnosine concentrations after an 8 week sprint interval program, most studies concerning this matter did not demonstrate positive effects after a 416 week isokinetic resistance training program (Kendrick et al., 2008; Kendrick et al., 2009; Mannion et al., 1994) or a 5 week sprint-training intervention (Baguet et al., 2011) on muscle carnosine concentrations in healthy subjects. The present study explored this in an animal MS model and confirmed that exercise therapy did not prevent reduction of muscle carnosine. Possibly, nutritional interventions (e.g. $\beta$-alanine supplementation) are required to exert such effects.

Muscular carnosine concentrations are a good marker of the total body carnosine stores, as $>99 \%$ of the compound is found in skeletal muscle cells (Boldyrev et al., 2013). A reduction of total carnosine stores is possibly also related to the increased oxidative stress and the resulting accumulation of cytotoxic compounds, such as the reactive carbonyls acrolein and hydroxynonenal (HNE). Carnosine serves as a sacrificial sequestering agent for acrolein and HNE by forming unreactive adducts (Song et al., 2014) and as such provides an endogenous protective mechanism against protein and ultimately 
tissue damage induced by these reactive carbonyls (Aldini et al., 2011; Baba et al., 2013). The increased urinary elimination of carnosine-carbonyl conjugates has been demonstrated for certain metabolic conditions (metabolic syndrome, obesity, type-2 diabetes) that are characterized by increased carbonyl stress (Regazzoni et al., 2016). Interestingly, acrolein has also been implicated in the pathogenesis of MS, as it directly damages myelin, through reaction with protein and lipid components of myelin, leading to demyelination. Furthermore, acrolein has recently been identified as a promising and effective therapeutic target (Leung et al., 2011; Tully and Shi, 2013) in this population. In fact, when Leung (Leung et al., 2011) and co-workers treated EAE mice with the acrolein scavenger hydralazine, myelin integrity appeared to be largely preserved indicating that acrolein removal may offer neuroprotection. Possibly, reduced muscle carnosine content in MS results from the incapacity of endogenous carnosine synthesis to compensate for the increased 'consumption' of carnosine to detoxify and eliminate acrolein. According to this line of reasoning, nutritional strategies to improve muscle carnosine content in persons with MS are worthwhile to explore. Carnosine supplementation ( $\sim 1.5 \mathrm{~g} / \mathrm{d} \beta$-alanine) has already been shown to improve a number of neurological symptoms (Boldyrev et al., 1987) in Alzheimer's (Preston et al., 1998) and Parkinson's (Boldyrev et al., 2013) disease. Clinically, nutritional interventions that normalize or even elevate the reduced muscle carnosine content could therefore be a valid new approach to improve muscle contractile properties, myocellular energy supply and/or possibly neurological symptoms in MS. Moreover, MS patients would be able to exercise more efficiently at higher intensities leading to a better clinical rehabilitation therapy outcome in these patients.

\section{Limitations}

The present study holds certain limitations that should be taken into account regarding future research. We show that EAE and MS reduce muscle carnosine, though because no nerve tissue was collected it was not possible to investigate whether this was due to neuroaxonal injury and/or muscle 
denervation. It is therefore warranted that in future EAE research nerve tissue is sampled to investigate peripheral nerves, motor end-plates and the extent of both neuronal and axonal damage in the spinal cord. In MS, future studies should also investigate spinal cord lesion load.

The current paper describes a reduction in muscle carnosine concentrations in an EAE group, compared to control animals. However, in order to determine whether reduced muscle carnosine levels were actually related to EAE itself, a control group with injection of Complete Freund's Adjuvant alone, causing inflammatory processes, would be appropriate. Indeed, the injection of CFA may have caused local inflammation and thus influenced $\mathrm{m}$. tibialis anterior carnosine concentrations. This issue may also be addressed by unilateral immunization and comparison of muscle carnosine of the two hind limbs. Furthermore, for future carnosine related research in MS, assessment of fatigue levels and dietary habits is worthwhile.

In summary, under the conditions of the present study EAE and MS reduce skeletal muscle carnosine levels. Exercise therapy alone could not prevent this in the animal MS model. This warrants further research investigating the effect of nutritional interventions that restore muscle carnosine levels either or not in combination with exercise therapy. 
Aldini, G., Orioli, M., Rossoni, G., Savi, F., Braidotti, P., Vistoli, G., Yeum, K.J., Negrisoli, G., Carini, M., 2011. The carbonyl scavenger carnosine ameliorates dyslipidaemia and renal function in Zucker obese rats. Journal of cellular and molecular medicine 15(6), 1339-1354.

Amorini, A.M., Nociti, V., Petzold, A., Gasperini, C., Quartuccio, E., Lazzarino, G., Di Pietro, V., Belli, A., Signoretti, S., Vagnozzi, R., Lazzarino, G., Tavazzi, B., 2014. Serum lactate as a novel potential biomarker in multiple sclerosis. Biochim Biophys Acta 1842(7), 1137-1143.

Baba, S.P., Hoetker, J.D., Merchant, M., Klein, J.B., Cai, J., Barski, O.A., Conklin, D.J., Bhatnagar, A., 2013. Role of aldose reductase in the metabolism and detoxification of carnosine-acrolein conjugates. The Journal of biological chemistry 288(39), 28163-28179.

Baguet, A., Everaert, I., De Naeyer, H., Reyngoudt, H., Stegen, S., Beeckman, S., Achten, E., Vanhee, L., Volkaert, A., Petrovic, M., Taes, Y., Derave, W., 2011. Effects of sprint training combined with vegetarian or mixed diet on muscle carnosine content and buffering capacity. Eur J Appl Physiol 111(10), 2571-2580.

Bellia, F., Vecchio, G., Rizzarelli, E., 2014. Carnosinases, their substrates and diseases. Molecules (Basel, Switzerland) 19(2), 2299-2329.

Blancquaert, L., Everaert, I., Derave, W., 2015. Beta-alanine supplementation, muscle carnosine and exercise performance. Curr Opin Clin Nutr Metab Care 18(1), 63-70.

Boldyrev, A.A., 2007. Carnosine and oxidative stress in cells and tissues. Nova Science Publishers, New York :.

Boldyrev, A.A., Aldini, G., Derave, W., 2013. Physiology and pathophysiology of carnosine. Physiol Rev 93(4), 1803-1845.

Boldyrev, A.A., Dupin, A.M., Bunin, A., Babizhaev, M.A., Severin, S.E., 1987. The antioxidative properties of carnosine, a natural histidine containing dipeptide. Biochem Int 15(6), 1105-1113. Campbell, G.R., Reeve, A.K., Ziabreva, I., Reynolds, R., Turnbull, D.M., Mahad, D.J., 2013. No excess of mitochondrial DNA deletions within muscle in progressive multiple sclerosis. Mult Scler 19(14), 18581866.

Constantinescu, C.S., Farooqi, N., O'Brien, K., Gran, B., 2011. Experimental autoimmune encephalomyelitis (EAE) as a model for multiple sclerosis (MS). Br J Pharmacol 164(4), 1079-1106. Dalgas, U., Stenager, E., Ingemann-Hansen, T., 2008. Multiple sclerosis and physical exercise: recommendations for the application of resistance-, endurance- and combined training. Mult Scler 14(1), 35-53.

Dawson, R., Jr., Biasetti, M., Messina, S., Dominy, J., 2002. The cytoprotective role of taurine in exercise-induced muscle injury. Amino Acids 22(4), 309-324.

de Haan, A., de Ruiter, C.J., van Der Woude, L.H., Jongen, P.J., 2000. Contractile properties and fatigue of quadriceps muscles in multiple sclerosis. Muscle Nerve 23(10), 1534-1541.

Derave, W., Everaert, I., Beeckman, S., Baguet, A., 2010. Muscle carnosine metabolism and betaalanine supplementation in relation to exercise and training. Sports Med 40(3), 247-263.

Derave, W., Ozdemir, M.S., Harris, R.C., Pottier, A., Reyngoudt, H., Koppo, K., Wise, J.A., Achten, E., 2007. beta-Alanine supplementation augments muscle carnosine content and attenuates fatigue during repeated isokinetic contraction bouts in trained sprinters. J Appl Physiol (1985) 103(5), 1736 1743.

Drozak, J., Piecuch, M., Poleszak, O., Kozlowski, P., Chrobok, L., Baelde, H.J., de Heer, E., 2015. UPF0586 Protein C9orf41 Homolog Is Anserine-producing Methyltransferase. The Journal of biological chemistry 290(28), 17190-17205.

Ellis, T., Motl, R.W., 2013. Physical activity behavior change in persons with neurologic disorders: overview and examples from Parkinson disease and multiple sclerosis. J Neurol Phys Ther 37(2), 8590.

Everaert, I., Stegen, S., Vanheel, B., Taes, Y., Derave, W., 2013. Effect of beta-alanine and carnosine supplementation on muscle contractility in mice. Med Sci Sports Exerc 45(1), 43-51. 
Garner, D.J., Widrick, J.J., 2003. Cross-bridge mechanisms of muscle weakness in multiple sclerosis. Muscle Nerve 27(4), 456-464.

Haider, L., Fischer, M.T., Frischer, J.M., Bauer, J., Hoftberger, R., Botond, G., Esterbauer, H., Binder, C.J., Witztum, J.L., Lassmann, H., 2011. Oxidative damage in multiple sclerosis lesions. Brain 134(Pt 7), 1914-1924.

Hansen, D., Wens, I., Vandenabeele, F., Verboven, K., Eijnde, B.O., 2015. Altered signaling for mitochondrial and myofibrillar biogenesis in skeletal muscles of patients with multiple sclerosis. Transl Res 166(1), 70-79.

Kendrick, I.P., Harris, R.C., Kim, H.J., Kim, C.K., Dang, V.H., Lam, T.Q., Bui, T.T., Smith, M., Wise, J.A., 2008. The effects of 10 weeks of resistance training combined with beta-alanine supplementation on whole body strength, force production, muscular endurance and body composition. Amino Acids 34(4), 547-554.

Kendrick, I.P., Kim, H.J., Harris, R.C., Kim, C.K., Dang, V.H., Lam, T.Q., Bui, T.T., Wise, J.A., 2009. The effect of 4 weeks beta-alanine supplementation and isokinetic training on carnosine concentrations in type I and II human skeletal muscle fibres. Eur J Appl Physiol 106(1), 131-138.

Kent-Braun, J.A., Ng, A.V., Castro, M., Weiner, M.W., Gelinas, D., Dudley, G.A., Miller, R.G., 1997.

Strength, skeletal muscle composition, and enzyme activity in multiple sclerosis. J Appl Physiol (1985) 83(6), 1998-2004.

Kent-Braun, J.A., Sharma, K.R., Miller, R.G., Weiner, M.W., 1994. Postexercise phosphocreatine resynthesis is slowed in multiple sclerosis. Muscle Nerve 17(8), 835-841.

Keytsman, C., Eijnde, B., Hansen, D., Verboven, K., Wens, I., 2017. Elevated cardiovasuclar risk factors in multiple sclerosis, Mult Scler Relat Disord. pp. 220-223.

Kjolhede, T., Vissing, K., Dalgas, U., 2012. Multiple sclerosis and progressive resistance training: a systematic review. Mult Scler 18(9), 1215-1228.

Kumleh, H.H., Riazi, G.H., Houshmand, M., Sanati, M.H., Gharagozli, K., Shafa, M., 2006. Complex I deficiency in Persian multiple sclerosis patients. J Neurol Sci 243(1-2), 65-69.

Leung, G., Sun, W., Zheng, L., Brookes, S., Tully, M., Shi, R., 2011. Anti-acrolein treatment improves behavioral outcome and alleviates myelin damage in experimental autoimmune encephalomyelitis mouse. Neuroscience 173, 150-155.

Mannion, A.F., Jakeman, P.M., Willan, P.L., 1994. Effects of isokinetic training of the knee extensors on high-intensity exercise performance and skeletal muscle buffering. European journal of applied physiology and occupational physiology 68(4), 356-361.

McCurdy, K., Langford, G., 2005. Comparison of unilateral squat strength between the dominant and non-dominant leg in men and women. Journal of sports science \& medicine 4(2), 153-159.

Motl, R.W., Gosney, J.L., 2008. Effect of exercise training on quality of life in multiple sclerosis: a meta-analysis. Mult Scler 14(1), 129-135.

Motl, R.W., Snook, E.M., Wynn, D.R., Vollmer, T., 2008. Physical activity correlates with neurological impairment and disability in multiple sclerosis. J Nerv Ment Dis 196(6), 492-495.

$\mathrm{Ng}$, A.V., Kent-Braun, J.A., 1997. Quantitation of lower physical activity in persons with multiple sclerosis. Med Sci Sports Exerc 29(4), 517-523.

Petajan, J.H., Gappmaier, E., White, A.T., Spencer, M.K., Mino, L., Hicks, R.W., 1996. Impact of aerobic training on fitness and quality of life in multiple sclerosis. Ann Neurol 39(4), 432-441.

Polfliet, M.M., van de Veerdonk, F., Dopp, E.A., van Kesteren-Hendrikx, E.M., van Rooijen, N., Dijkstra, C.D., van den Berg, T.K., 2002. The role of perivascular and meningeal macrophages in experimental allergic encephalomyelitis. J Neuroimmunol 122(1-2), 1-8.

Preston, J.E., Hipkiss, A.R., Himsworth, D.T., Romero, I.A., Abbott, J.N., 1998. Toxic effects of betaamyloid(25-35) on immortalised rat brain endothelial cell: protection by carnosine, homocarnosine and beta-alanine. Neurosci Lett 242(2), 105-108.

Pugliatti, M., Rosati, G., Carton, H., Riise, T., Drulovic, J., Vecsei, L., Milanov, I., 2006. The epidemiology of multiple sclerosis in Europe. Eur J Neurol 13(7), 700-722.

Regazzoni, L., de Courten, B., Garzon, D., Altomare, A., Marinello, C., Jakubova, M., Vallova, S., Krumpolec, P., Carini, M., Ukropec, J., Ukropcova, B., Aldini, G., 2016. A carnosine intervention study 
in overweight human volunteers: bioavailability and reactive carbonyl species sequestering effect. Scientific reports 6, 27224.

Rice, C.L., Vollmer, T.L., Bigland-Ritchie, B., 1992. Neuromuscular responses of patients with multiple sclerosis. Muscle Nerve 15(10), 1123-1132.

Sallis, J.F., Haskell, W.L., Fortmann, S.P., Wood, P.D., Vranizan, K.M., 1986. Moderate-intensity physical activity and cardiovascular risk factors: the Stanford Five-City Project. Preventive medicine 15(6), 561-568.

Savci, S., Inal-Ince, D., Arikan, H., Guclu-Gunduz, A., Cetisli-Korkmaz, N., Armutlu, K., Karabudak, R., 2005. Six-minute walk distance as a measure of functional exercise capacity in multiple sclerosis. Disabil Rehabil 27(22), 1365-1371.

Schulz, K.H., Gold, S.M., Witte, J., Bartsch, K., Lang, U.E., Hellweg, R., Reer, R., Braumann, K.M., Heesen, C., 2004. Impact of aerobic training on immune-endocrine parameters, neurotrophic factors, quality of life and coordinative function in multiple sclerosis. J Neurol Sci 225(1-2), 11-18.

Sharma, K.R., Kent-Braun, J., Mynhier, M.A., Weiner, M.W., Miller, R.G., 1995. Evidence of an abnormal intramuscular component of fatigue in multiple sclerosis. Muscle Nerve 18(12), 1403-1411. Siqueira, C.M., Pelegrini, F.R., Fontana, M.F., Greve, J.M., 2002. Isokinetic dynamometry of knee flexors and extensors: comparative study among non-athletes, jumper athletes and runner athletes. Revista do Hospital das Clinicas 57(1), 19-24.

Song, B.C., Joo, N.S., Aldini, G., Yeum, K.J., 2014. Biological functions of histidine-dipeptides and metabolic syndrome. Nutrition research and practice 8(1), 3-10.

Stuerenburg, H.J., Kunze, K., 1999. Concentrations of free carnosine (a putative membraneprotective antioxidant) in human muscle biopsies and rat muscles. Archives of gerontology and geriatrics 29(2), 107-113.

Stuifbergen, A.K., Blozis, S.A., Harrison, T.C., Becker, H.A., 2006. Exercise, functional limitations, and quality of life: A longitudinal study of persons with multiple sclerosis. Arch Phys Med Rehabil 87(7), 935-943.

Suzuki, Y., 2004. The Effect of Sprint Training on Skeletal Muscle Carnosine in Humans. Int J Sport Health Sci 2, 105-110.

Tallon, M.J., Harris, R.C., Maffulli, N., Tarnopolsky, M.A., 2007. Carnosine, taurine and enzyme activities of human skeletal muscle fibres from elderly subjects with osteoarthritis and young moderately active subjects. Biogerontology 8(2), 129-137.

Tully, M., Shi, R., 2013. New insights in the pathogenesis of multiple sclerosis--role of acrolein in neuronal and myelin damage. Int J Mol Sci 14(10), 20037-20047.

van der Kamp, W., Maertens de Noordhout, A., Thompson, P.D., Rothwell, J.C., Day, B.L., Marsden, C.D., 1991. Correlation of phasic muscle strength and corticomotoneuron conduction time in multiple sclerosis. Ann Neurol 29(1), 6-12.

Wassif, W.S., Sherwood, R.A., Amir, A., Idowu, B., Summers, B., Leigh, N., Peters, T.J., 1994. Serum carnosinase activities in central nervous system disorders. Clinica chimica acta; international journal of clinical chemistry 225(1), 57-64.

Wens, I., Dalgas, U., Vandenabeele, F., Grevendonk, L., Verboven, K., Hansen, D., Eijnde, B.O., 2015a. High Intensity Exercise in Multiple Sclerosis: Effects on Muscle Contractile Characteristics and Exercise Capacity, a Randomised Controlled Trial. PLoS One 10(9), e0133697.

Wens, I., Dalgas, U., Vandenabeele, F., Krekels, M., Grevendonk, L., Eijnde, B.O., 2014. Multiple sclerosis affects skeletal muscle characteristics. PLoS One 9(9), e108158.

Wens, I., Dalgas, U., Verboven, K., Kosten, L., Stevens, A., Hens, N., Eijnde, B.O., 2015b. Impact of high intensity exercise on muscle morphology in EAE rats. Physiol Res 64(6), 907-923. 
